TRANSACTIONS OF THE

AMERICAN MATHEMATICAL SOCIETY

Volume 364, Number 11, November 2012, Pages 5791-5812

S 0002-9947(2012)05755-2

Article electronically published on June 8, 2012

\title{
ANALYSIS ON SOME INFINITE MODULES, INNER PROJECTION, AND APPLICATIONS
}

\author{
KANGJIN HAN AND SIJONG KWAK
}

Dedicated to the memory of Hyo Chul Myung (June, 1937-February, 2010)

\begin{abstract}
A projective scheme $X$ is called 'quadratic' if $X$ is scheme-theoretically cut out by homogeneous equations of degree 2 . Furthermore, we say that $X$ satisfies 'property $\mathbf{N}_{2, p}$ ' if it is quadratic and the quadratic ideal has only linear syzygies up to the first $p$-th steps. In the present paper, we compare the linear syzygies of the inner projections with those of $X$ and obtain a theorem on 'embedded linear syzygies' as one of our main results. This is the natural projection-analogue of 'restricting linear syzygies' in the linear section case. As an immediate corollary, we show that the inner projections of $X$ satisfy property $\mathbf{N}_{2, p-1}$ for any reduced scheme $X$ with property $\mathbf{N}_{2, p}$.

Moreover, we also obtain the neccessary lower bound $(\operatorname{codim} X) \cdot p-\frac{p(p-1)}{2}$, which is sharp, on the number of quadrics vanishing on $X$ in order to satisfy $\mathbf{N}_{2, p}$ and show that the arithmetic depths of inner projections are equal to that of the quadratic scheme $X$. These results admit an interesting 'syzygetic' rigidity theorem on property $\mathbf{N}_{2, p}$ which leads the classifications of extremal and next-to-extremal cases.

For these results we develop the elimination mapping cone theorem for infinitely generated graded modules and improve the partial elimination ideal theory initiated by M. Green. This new method allows us to treat a wider class of projective schemes which cannot be covered by the Koszul cohomology techniques because these are not projectively normal in general.
\end{abstract}

\section{Contents}

Introduction

1. Definitions and preliminaries

2. Elimination mapping cone construction and partial

elimination ideals

3. Embedded linear syzygies and applications

4. Arithmetic depth and syzygetic rigidity

5. Examples and open questions

Acknowledgements

References

Received by the editors October 14, 2010.

2010 Mathematics Subject Classification. Primary 14N05, 13D02, 14N25; Secondary 51N35.

Key words and phrases. Linear syzygies, the mapping cone theorem, partial elimination ideals, inner projection, arithmetic depth, Castelnuovo-Mumford regularity.

The authors were supported by the Basic Science Research Program through the National Research Foundation of Korea (NRF) funded by the Ministry of Education, Science and Technology (grant No. 2009-0063180). 


\section{INTRODUCTION}

Let $X$ be a nondegenerate reduced closed subscheme in a projective space $\mathbb{P}^{N}$ over an algebraically closed field $k$ of characteristic zero and $R=k\left[x_{0}, \ldots, x_{N}\right]$ be the coordinate ring of $\mathbb{P}^{N}$. The equations defining $X$ and the syzygies among them have played a central role to study projective schemes in algebraic geometry. Further the syzygy structures and their geometric implications have been intensively focused for the most interesting case, i.e. projective schemes having property $\mathbf{N}_{2, p}$; for the last twenty years, see CKK06, EGHP05, EGHP06, EHU06, GL88. They are closely related to the Eisenbud-Goto conjecture on Castelnuovo-Mumford regularity and other conjectures on linear syzygies in classical algebraic geometry. The linear sections and projections of $X$ have been very useful to understand those problems.

For the linear sections, we have interesting results on 'Restricting linear syzygies' due to Eisenbud, Green, Hulek, and Popescu; see [EGHP05. Along this line, a natural question could be raised:

What is the relation between the syzygies of projections and $X$ ?

In the present paper, we especially consider the relations between the linear syzygies of inner projections and those of $X$. Note that the inner projection has been a standard issue classically since del Pezzo and Fano used this projection for the classification of del Pezzo surfaces and Fano 3-folds; see Reid00. There are also some known results about nonbirational loci of these projection morphisms and geometric structures of the projection images; see [BHSS00, CC01, Seg36, Som79].

Problems. We list our main problems in detail:

(a) (Embedded linear syzygies) Let $X$ be a nondegenerate reduced scheme in $\mathbb{P}^{N}$ satisfying property $\mathbf{N}_{2, p}(p \geq 1)$. Consider the linear projection from a linear subvariety $\Lambda \subset \mathbb{P}^{N}$ of $\operatorname{dim} \Lambda=t<p$ with $\Lambda \cap X \neq \emptyset,\langle\Lambda \cap X\rangle=$ $\Lambda$ and $X_{\Lambda}=\overline{\pi_{\Lambda}(X \backslash \Lambda)}$ in $\mathbb{P}^{N-t-1}$. How do the syzygies behave under projections? D. Eisenbud et al. showed that, under some $\mathbf{N}_{2, p}$-assumption, the syzygies of $X$ restrict surjectively to the syzygies of linear sections in their paper 'Restricting linear syzygies', EGHP05]. Is there any natural projection-analogue of the linear section case? Bearing on this problem, we also expect that $X_{\Lambda}$ satisfies property $\mathbf{N}_{2, p-t-1}$.

(b) (Necessary lower bound for property $\mathbf{N}_{2, p}$ ) For a quadratic scheme $X$ satisfying $\mathbf{N}_{2, p}$, it is roughly believed that the more quadratic equations $X$ has, the further steps linear syzygies proceed to. Therefore one can ask 'how many quadrics does $X$ require to satisfy property $\mathbf{N}_{2, p}$ ?' This is a natural question, but not yet known.

(c) (Syzygetic rigidity theorem) In EGHP05, EGHP06 they also show that a closed subscheme $X \subset \mathbb{P}^{N}$ is 2-regular if $X$ satisfies property $\mathbf{N}_{2, \operatorname{codim} X}$ and characterize all 2-regular algebraic sets geometrically for this extremal case. What about the 'next-to-extremal case', i.e. a scheme $X$ satisfying $\mathbf{N}_{2, \operatorname{codim} X-1}$ ? How do we classify or characterize them in a suitable category?

For those problems, we develop the elimination mapping cone theorem for infinitely generated graded modules and improve the partial elimination ideal theory initiated by M. Green for the inner projection. This allows us to treat a wider class of projective schemes which cannot be covered by the Koszul cohomology techniques because these are not projectively normal in general. We have also found it 
very interesting to understand some relations between the syzygies of its projections and those of $X$ as we move the center of projection.

Organization of the paper. We recall basic definitions and preliminaries in Section 1. In Section 2, we set up the elimination mapping cone construction for infinitely generated graded modules and the partial elimination ideal theory for inner projection which are crucial to understand the syzygy structures of infinitely generated graded modules. This partial elimination ideal theory gives us local information on $X$ near the center of projection $q \in X$ which turns out to govern syzygies and other properties of the inner projection $X_{q}$ from the (global) homogeneous equations.

In Section 3, we obtain some results on syzygy structures and geometric properties of inner projections, i.e. embedded linear syzygies, the number of quadratic equations, and their corollaries. In particular, we can show that for any projective reduced scheme $X$ satisfying property $\mathbf{N}_{2, p}$ the inner projection from any smooth point satisfies at least property $\mathbf{N}_{2, p-1}$ and $X_{\Lambda}$ satisfies at least $\mathbf{N}_{2, p-t-1}$ for a general $t$-dimensional linear subspace $\Lambda$ with $\operatorname{dim} X \cap \Lambda=0$ (see Corollary 3.4 and Remark 3.5). We also give the neccessary lower bound $(\operatorname{codim} X) \cdot p-\frac{p(p-1)}{2}$ on the number of quadrics vanishing on $X$ in order to satisfy property $\mathbf{N}_{2, p}$, which is sharp.

In Section 4, we prove that the arithmetic depths of inner projections are equal to those of the given quadratic scheme. Combined with results in the previous section, this depth theorem leads us to a very interesting 'syzygetic' rigidity theorem on property $\mathbf{N}_{2, p}$ in the category of varieties; namely, for the extremal (i.e. $p=$ $\operatorname{codim} X$ ) and next-to-extremal (i.e. $p=\operatorname{codim} X-1$ ) cases those varieties should be arithmetically Cohen-Macaulay (abbr. ACM) and we can give the classfications of the two cases. We also extend this result to a more general category (See Corollary 4.5 and Question [5.6). Finally, in Section 5, we see some examples and open questions stimulating further work.

\section{Definitions AND PRELIMINARIES}

We work over an algebraically closed field $k$ of characteristic zero. Let $X$ be a nondegenerate reduced closed subscheme in a projective space $\mathbb{P}^{N}$.

Definition 1.1. Let $X$ be as above.

(a) $X$ is said to be a quadratic scheme if there is a homogeneous ideal $I$ generated by equations of degree 2 which defines $X$ scheme-theoretically (i.e. its sheafification $\widetilde{I}$ is equal to the ideal sheaf $\mathcal{J}_{X}$ of $X$ ).

(b) $X$ is said to satisfy property $\mathbf{N}_{2, p}$ scheme-theoretically if it is quadratic and the quadratic ideal $I$ has only linear syzygies at least up to the first $p$-th steps.

(c) $X$ is said to be $m$-regular if $H^{i}\left(\mathcal{J}_{X}(m-i)\right)=0$ for all $i \geq 1$. We call $\operatorname{reg}(X):=\min \left\{m \mid H^{i}\left(\mathcal{J}_{X}(m-i)\right)=0\right.$ for all $\left.i \geq 1\right\}$ Castelnuovo-Mumford regularity of $X$.

Note that Definition 1.1 (b) is a generalization of known notions. It is the same as property $\mathbf{N}_{p}$ defined by Green-Lazarsfeld if $X$ is projectively normal and $I$ is saturated (see GL88). 
Let $\pi_{\Lambda}: X \subset \mathbb{P}^{N} \rightarrow \mathbb{P}^{N-t-1}$ denote the projection of $X$ from a linear space $\Lambda=\mathbb{P}^{t}$. We call it either an outer projection if $X \cap \Lambda=\emptyset$ or an inner projection in case $\Lambda \subset X$. Every projection $\pi_{\Lambda}$ can be regarded as succesive compositions of suitable outer and inner projections from points. These projections as well as blowups have been very useful projective techniques in algebraic geometry. We briefly review the preliminaries about an inner projection from a point $q \in X$.

Let $\sigma: \widetilde{X} \rightarrow X$ be a blowing up of $X$ at a smooth point $q \in X$. One has the regular morphism $\pi^{\prime}: \widetilde{X} \rightarrow X_{q}:=\overline{\pi_{q}(X \backslash\{q\})} \subset \mathbb{P}^{N-1}$ with the following diagram:

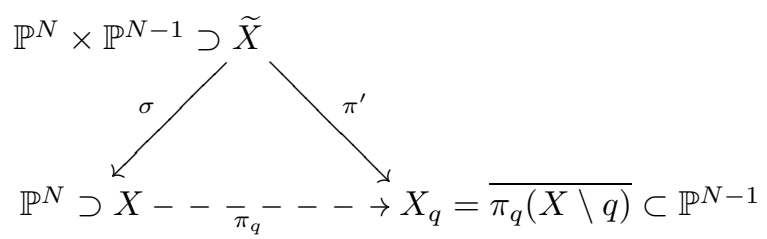

Classically, one says that a smooth variety $X$ admits an inner projection if $\pi^{\prime}$ is an embedding for some point $q \in X$. This is equivalent to $q \in X \backslash \operatorname{Trisec}(X)$ where $\operatorname{Trisec}(X)$ is the union of all lines $\ell$ with the condition that $\ell \subset X$ or $X \cap \ell$ is a subscheme of length at least 3 . We also know that the exceptional divisor $E$ is linearly embedded via $\pi^{\prime}$ in $\mathbb{P}^{N-1}$ (i.e. $\pi^{\prime}(E)=\mathbb{P}^{r-1} \subset \mathbb{P}^{N-1}, r=\operatorname{dim} X$ ) for any subvariety $X$ if the center $q$ is smooth (see Bau95, FCV99]).

Let $R=k\left[x_{0}, \ldots, x_{N}\right]$ and $S=k\left[x_{1}, x_{2}, \ldots, x_{N}\right]$ be the homogeneous coordinate rings of $\mathbb{P}^{N}$ and $\mathbb{P}^{N-1}$. Assume $q=(1: 0: \ldots: 0) \in X$ (by suitable coordinate change). Let $I$ be an ideal of $R$ defining a reduced scheme $X$ scheme-theoretically. Naturally we can give a scheme structure on the image $X_{q}$ by the ideal $J:=I \cap S$. Note that the ideal $J$ is reduced if $I$ is reduced.

In the case of inner projection we note that $R / I$ is not a finitely generated $S$-module because $q \in X$ and there is no polynomial of the form $f=x_{0}{ }^{n}+$ (other terms) for some $n \in \mathbb{N}$ in the ideal $I$, even though $R / I$ is finitely generated as an $R$-module. $I$ is also an infinitely generated graded $S$-module with the following resolution:

$$
\cdots \rightarrow \bigoplus_{j=2}^{\infty} S(-i-j)^{\beta_{i, j}} \rightarrow \cdots \rightarrow \bigoplus_{j=2}^{\infty} S(-j)^{\beta_{0, j}} \rightarrow I \rightarrow 0 .
$$

In Section 2, we show that they have interesting syzygy structures as $S$-modules (see Proposition 2.5 and Remark 2.6).

On the other hand, if $X$ is quadratic, then we can write the quadratic ideal $I$ as

$$
I=\left(x_{0} \ell_{1}-Q_{0,1}, \ldots, x_{0} \ell_{t}-Q_{0, t}, Q_{1}, \ldots, Q_{s}\right), q=(1,0, \ldots, 0) \in X,
$$

where $\ell_{i}$ is a linear form, $Q_{0, i}, Q_{j}$ are quadratic forms in $S=k\left[x_{1}, \ldots, x_{N}\right]$ and they are minimal generators. We can also assume that all $\left\{\ell_{i}\right\}$ are linearly independent, and all $\left\{Q_{0, i}\right\}$ are distinct. Clearly, $\left\{\ell_{i}\right\}$ generate $\left(T_{q} X\right)^{*}$. Note that $t=\operatorname{codim}(X)=N-\operatorname{dim} X$ if $q$ is a smooth point. In general, $t$ is equal to $N-\operatorname{dim} T_{q} X$

Convention. We are working on the following convention:

- Let $X \subset \mathbb{P}^{N}$ and $q \in X$ be as above and $I$ be a homogeneous defining ideal of $X$. We denote the $S$-ideal $I \cap S$ by $J$ which gives the natural induced scheme structure on the projection image $X_{q} \subset \mathbb{P}^{N-1}$ and call $J$ the $x_{0}$ elimination ideal of $I$. In addition, we write the saturated ideal defining $X$ (resp. $X_{q}$ ) as $I_{X}\left(\operatorname{resp} . I_{X_{q}}\right)$. 
- (Betti numbers) For a graded $R$-module $M$, we define graded Betti numbers $\beta_{i, j}^{R}(M)$ of $M$ by $\operatorname{dim}_{k} \operatorname{Tor}_{i}^{R}(M, k)_{i+j}$. We consider $\beta_{i, j}^{S}(N)$ for any graded $S$-module $N$ in the same manner. We remind readers that $\operatorname{Tor}_{i}^{R}(R / I, k)_{i+j}$ $=\operatorname{Tor}_{i-1}^{R}(I, k)_{i-1+j+1}$. So $\beta_{i, j}^{R}(R / I)=\beta_{i-1, j+1}^{R}(I)$. We write $\beta_{i, j}^{R}(M)$ as $\beta_{i, j}(M)$ or $\beta_{i, j}$ if it is obvious.

- We often abbreviate $\operatorname{Tor}_{i}^{R}(M, k)_{i+j}$ as $\operatorname{Tor}_{i}^{R}(M)_{i+j}$ (same for $S$-module Tor).

- (Arithmetic depth) When we refer to the depth of $X$, denoted by $\operatorname{depth}_{R}(X)$, we mean the arithmetic depth of $X$, i.e. $\operatorname{depth}_{R}\left(R / I_{X}\right)$.

\section{Elimination MAPPING CONE CONSTRUCTION AND PARTIAL ELIMINATION IDEALS}

In general the mapping cone of the chain map between two complexes is a kind of natural extension of complexes induced by the given chain map. Now we construct some graded mapping cone which we call the 'elimination mapping cone'. This is naturally related to projections and very useful to understand the syzygies of projections. Another ingredient is the partial elimination ideal theory. Let us construct the graded mapping cone theorem and consider the partial elimination ideal theory from the viewpoint of inner projections.

Elimination mapping cone construction. Let $W=k\left\langle x_{1}, \ldots, x_{N}\right\rangle \subset V=$ $k\left\langle x_{0}, \ldots, x_{N}\right\rangle$ be vector spaces over $k$ and $S=\operatorname{Sym}(W)=k\left[x_{1}, \ldots, x_{N}\right] \subset R=$ $\operatorname{Sym}(V)=k\left[x_{0}, \ldots, x_{N}\right]$ be polynomial rings.

- $M$ : a graded $R$-module given a degree 1 shifting map by $\mu$

(i.e. $\mu: M_{i} \rightarrow M_{i+1}$ )

- $\mathbb{G}_{*}\left(\right.$ resp. $\left.\mathbb{F}_{*}\right)$ : the graded Koszul complex of $M, K_{*}^{S}(M)$

(resp. $\left.M[-1], K_{*}^{S}(M[-1])\right)$ as follows:

$$
0 \rightarrow \wedge^{N} W \otimes M \rightarrow \cdots \rightarrow \wedge^{2} W \otimes M \rightarrow W \otimes M \rightarrow M \rightarrow 0
$$

whose graded components $\left(\mathbb{G}_{i}\right)_{i+j}$ are $K_{i}^{S}(M)_{i+j}=\wedge^{i} W \otimes M_{j}$ (resp. $\left.\left(\mathbb{F}_{i}\right)_{i+j}=\wedge^{i} W \otimes M_{j-1}\right)$.

- Then $\mu: M_{i} \rightarrow M_{i+1}$ induces the chain map $\bar{\mu}: \mathbb{F}_{*}=K_{*}^{S}(M[-1]) \longrightarrow \mathbb{G}_{*}=K_{*}^{S}(M)$ of degree 0.

Now we construct the mapping cone $\left(\mathbb{C}_{\bar{\mu}}, \mathrm{d}_{\bar{\mu}}\right)$ such that

$$
0 \longrightarrow \mathbb{G}_{*} \longrightarrow\left(\mathbb{C}_{\bar{\mu}}\right)_{*} \longrightarrow \mathbb{F}_{*}[-1] \longrightarrow 0,
$$

where $\mathbb{C}_{\bar{\mu}}$ is a direct sum $\mathbb{G}_{*} \oplus \mathbb{F}_{*}[-1]$ and the differential $\mathrm{d}_{\bar{\mu}}$ is given by

$$
\left(\mathrm{d}_{\bar{\mu}}\right)_{*}=\left(\begin{array}{cc}
\partial_{\mathbb{G}} & (-1)^{*+1} \bar{\mu} \\
0 & \partial_{\mathbb{F}}
\end{array}\right),
$$

where $\partial$ is the differential of the Koszul complex. From the construction, it can be checked that we have the isomorphism (see AK11):

$$
\operatorname{Tor}_{i}^{R}(M)_{i+j} \simeq H_{i}\left(\left(\mathbb{C}_{\bar{\mu}}\right)_{*}\right)_{i+j} .
$$

Suppose $M$ is a graded $R$-module which is also a graded $S$-module. Consider a multiplication map $\mu=\times x_{0}$ as a naturally given degree 1 shifting map on $M$. In this case, the long exact sequence on homology groups induced from (2.1) is important and very useful to study the syzygies of projections. Note that in general we can define property $\boldsymbol{N}_{d, p}$ similarly (i.e. $\beta_{i, j}^{R}(R / I)=0$ for any $0 \leq i \leq p, j \geq d$ ). 
Theorem 2.1 (Elimination mapping cone sequence). Let $S=k\left[x_{1}, \ldots, x_{N}\right] \subset R=$ $k\left[x_{0}, x_{1}, \ldots, x_{N}\right]$ be two polynomial rings.

(a) Let $M$ be a graded $R$-module which is not necessarily finitely generated. Then, we have a natural long exact sequence:

$$
\cdots \operatorname{Tor}_{i}^{R}(M)_{i+j} \rightarrow \operatorname{Tor}_{i-1}^{S}(M)_{i-1+j} \stackrel{\bar{\mu}}{\rightarrow} \operatorname{Tor}_{i-1}^{S}(M)_{i-1+j+1} \rightarrow \operatorname{Tor}_{i-1}^{R}(M)_{i-1+j+1} \cdots
$$
whose connecting homomorphism $\bar{\mu}$ is induced by the multiplicative map $\times x_{0}$.

(b) Assume that $R / I$ satisfies property $\boldsymbol{N}_{d, p}$ for some $d \geq 2, p \geq 1$. Then a multiplication by $x_{0}$ induces a sequence of isomorphisms on $\operatorname{Tor}_{i}^{S}(I)_{i+j}$ for $0 \leq i \leq p-2, j \geq d+1$ and a surjection for $j=d$ :

$$
\cdots \stackrel{\times x_{0}}{\rightarrow} \operatorname{Tor}_{i}^{S}(I)_{i+d} \stackrel{\times x_{0}}{\rightarrow} \operatorname{Tor}_{i}^{S}(I)_{i+d+1} \stackrel{\times x_{0}}{\stackrel{\sim}{\rightarrow}} \operatorname{Tor}_{i}^{S}(I)_{i+d+2} \stackrel{\times x_{0}}{\rightarrow} \cdots .
$$

For $i=p-1$, we have a sequence of surjections from $j=d$ :

$$
\cdots \stackrel{\times x_{0}}{\rightarrow} \operatorname{Tor}_{p-1}^{S}(I)_{p-1+d} \stackrel{\times x_{0}}{\rightarrow} \operatorname{Tor}_{p-1}^{S}(I)_{p-1+d+1} \stackrel{\times x_{0}}{\rightarrow} \operatorname{Tor}_{p-1}^{S}(I)_{p-1+d+2} \stackrel{\times x_{0}}{\rightarrow} \cdots .
$$

Remark 2.2. J. Ahn and the second author pointed out that this graded mapping cone construction is closely related to outer projections (see AK11]). We remark here that this theorem is also true for an infinitely generated $S$-module $M$ and relates the torsion module $\operatorname{Tor}^{R}(M)$ to the torsion module of $M$ as an $S$-module. Therefore this gives us useful information about syzygies of inner projections.

Proof. (a) follows from theorem 2.2 in AK11. For a proof of (b), consider the mapping cone sequence of Theorem 2.1 for $M=I$ :

$$
\operatorname{Tor}_{i+1}^{R}(I)_{i+1+j} \rightarrow \operatorname{Tor}_{i}^{S}(I)_{i+j} \stackrel{\times x_{0}}{\longrightarrow} \operatorname{Tor}_{i}^{S}(I)_{i+j+1} \rightarrow \operatorname{Tor}_{i}^{R}(I)_{i+j+1} .
$$

Note that $\operatorname{Tor}_{i}^{R}(I, k)_{i+j}=0$ for $0 \leq i \leq p-1$ and $j \geq d+1$ by the assumption that $I$ has the $\mathbf{N}_{d, p}$ property as an $R$-module. So, we have an isomorphism

$$
\operatorname{Tor}_{i}^{S}(I, k)_{i+j} \stackrel{\times x_{0}}{\rightarrow} \operatorname{Tor}_{i}^{S}(I, k)_{i+j+1}
$$

for $0 \leq i \leq p-2, \forall j \geq d+1$ and a surjection for $j=d$.

In the case $i=p-1$, we know that $\operatorname{Tor}_{p-1}^{R}(I)_{p-1+j}=0$ for $j \geq d+1$ in the mapping cone sequence

$$
\operatorname{Tor}_{p}^{R}(I)_{p+j} \rightarrow \operatorname{Tor}_{p-1}^{S}(I)_{p-1+j} \stackrel{\times x_{0}}{\longrightarrow} \operatorname{Tor}_{p-1}^{S}(I)_{p-1+j+1} \rightarrow \operatorname{Tor}_{p-1}^{R}(I)_{p-1+j+1} .
$$

Therefore we get the desired surjections for the $i=p-1$ case.

Partial elimination ideals under a projection. Mark Green introduced partial elimination ideals in his lecture note Gre98. For the degree lexicographic order, if $f \in I_{m}$ has leading term $\operatorname{in}(f)=x_{0}^{d_{0}} \cdots x_{n}^{d_{n}}$, we set $d_{0}(f)=d_{0}$, the leading power of $x_{0}$ in $f$. Then we can give the definition of partial elimination ideals as in the following.

Definition 2.3. Let $I \subset R$ be a homogeneous ideal and let

$$
\widetilde{K}_{i}(I)=\bigoplus_{m \geq 0}\left\{f \in I_{m} \mid d_{0}(f) \leq i\right\} .
$$


If $f \in \widetilde{K}_{i}(I)$, we may write uniquely $f=x_{0}^{i} \bar{f}+g$, where $d_{0}(g)<i$. Now we define the ideal $K_{i}(I)$ in $S$ generated by the image of $\widetilde{K}_{i}(I)$ under the map $f \mapsto \bar{f}$ and we call $K_{i}(I)$ the $i$-th partial elimination ideal of $I$.

Observation 2.4. We can observe some properties of these ideals in the projection case.

(a) The 0 -th partial elimination ideal $K_{0}(I)$ of $I$ is

$$
J:=I \cap S=\bigoplus_{m \geq 0}\left\{f \in I_{m} \mid d_{0}(f)=0\right\} .
$$

Note that the ideal $J$ gives a scheme structure on the image $X_{q}$ naturally.

(b) $\widetilde{K}_{i}(I)$ is a natural filtration of $I$ with respect to $x_{0}$, which also induces a filtraton on $K_{i}(I)$ 's :

$$
\begin{gathered}
J=\widetilde{K}_{0}(I) \subset \widetilde{K}_{1}(I) \subset \cdots \subset \widetilde{K}_{i}(I) \subset \cdots \subset \widetilde{K}_{\infty}(I)=I, \\
J=K_{0}(I) \subset K_{1}(I) \subset \cdots \subset K_{i}(I) \subset \cdots \subset S .
\end{gathered}
$$

(c) $\widetilde{K}_{i}(I)$ is a finitely generated graded $S$-module and there is a short exact sequence as graded $S$-modules:

$$
0 \rightarrow \frac{\widetilde{K}_{i-1}(I)}{\widetilde{K}_{0}(I)} \rightarrow \frac{\widetilde{K}_{i}(I)}{\widetilde{K}_{0}(I)} \rightarrow K_{i}(I)(-i) \rightarrow 0 .
$$

In general we can at least see when the $K_{i}(I)$ 's stabilize and what they look like for inner projections. The following proposition is the anwser. It also tells us a minimal free resoultion for some infinitely generated graded $S$-module which is very useful to understand the defining equations and syzygies of inner projections.

Proposition 2.5. Let $X \subset \mathbb{P}^{N}$ be a reduced projective scheme with a homogeneous defining ideal $I$. Let $q=(1,0, \ldots, 0) \in X$.

(a) If I satisfies property $\boldsymbol{N}_{d, 1}, K_{i}(I)$ stabilizes at least at $i=d-1$ to an ideal defining $T C_{q} X$, the tangent cone of $X$ at $q$. So if $q$ is smooth, $K_{d-1}(I)$ consists of linear forms which define $T_{q} X$.

(b) In particular, if $I$ is generated by quadrics and $q$ is smooth, then $K_{i}(I)$ stabilizes at the $i=1$ step to an ideal $I_{L}=\left(l_{1}, \ldots, l_{e}\right), e=\operatorname{codim}\left(X, \mathbb{P}^{N}\right)$ which defines the tangent space $T_{q} X$, i.e. $J=K_{0}(I) \subset I_{L}=K_{1}(I)=$ $\cdots=K_{i}(I)=\cdots \subset S$ and $I / J$ has obvious syzygies as an infinitely generated $S$-module such that:

$$
\begin{aligned}
& S(-e-1)^{b_{e}} \quad S(-3)^{b_{2}} \quad S(-2)^{b_{1}}
\end{aligned}
$$

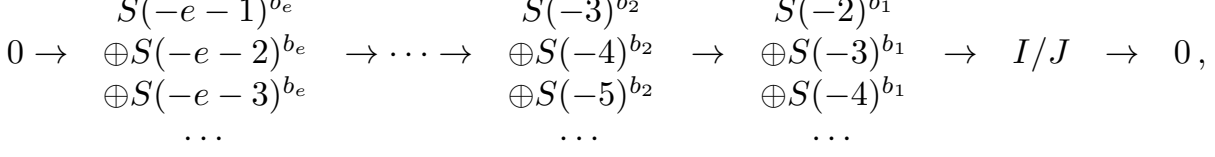

where $b_{i}=\left(\begin{array}{l}e \\ i\end{array}\right)$.

Proof. (a) Since $I$ is generated in $\operatorname{deg} \leq d$ and $q=(1,0, \ldots, 0) \in X$, we have generators $\left\{F_{i}\right\}$ of $I$ with $d_{0}\left(F_{i}\right) \leq d-1$ because there is no generator of the form $x_{0}{ }^{d}+$ other lower terms in $x_{0}$. From this, every leading term $f$ of a homogeneous polynomial $F$ in $I$ of $\operatorname{deg} k(k \geq d)$ is written as $x_{0}^{\square} \cdot \bar{f}$, where $\bar{f} \in K_{c}(I)$ for some $c \leq d-1$. So $K_{i}(I)$ stabilizes at least at $i=d-1$. Note that all $\bar{f} \in K_{i}(I)(i \geq 0)$ are also regarded as the defining equations of the tangent cone $T C_{q} X$ of $X$ at $q$ because they come from $f=x_{0}^{i} \bar{f}+g \in I, d_{0}(g)<i$. Therefore, $K_{i}(I)$ stabilizes to 
the ideal defining $T C_{q} X$. In the case of a smooth point $q \in X, T_{q} X=T C_{q} X$ and $K_{d-1}(I)=\left(\ell_{1}, \ldots, \ell_{e}\right), e=\operatorname{codim}\left(X, \mathbb{P}^{N}\right)$.

(b) Since $d=2$ and $q$ is a smooth point, $K_{i}(I)$ becomes $I_{L}=\left(\ell_{1}, \ldots, \ell_{e}\right)$ for each $i \geq 1$. For the sake of the $S$-module syzygy of $I / J$, first note that $I=\widetilde{K}_{\infty}(I)$. From the exact sequence (2.2), we get $\widetilde{K}_{1}(I) / J \simeq K_{1}(I)(-1)$ with the following linear Koszul resolution: letting $b_{i}=\left(\begin{array}{l}e \\ i\end{array}\right)$,

$$
0 \rightarrow S(-e-1)^{b_{e}} \rightarrow \cdots \rightarrow S(-3)^{b_{2}} \rightarrow S(-2)^{b_{1}} \rightarrow \widetilde{K}_{1}(I) / J \rightarrow 0 .
$$

Next, $K_{2}(I)(-2)=K_{1}(I)(-2)$ also has linear syzygies:

$$
0 \rightarrow S(-e-2)^{b_{e}} \rightarrow \cdots \rightarrow S(-4)^{b_{2}} \rightarrow S(-3)^{b_{1}} \rightarrow K_{2}(I)(-2) \rightarrow 0,
$$

and we have the following exact sequence from (2.2) again:

$$
0 \rightarrow \frac{\widetilde{K}_{1}(I)}{J} \rightarrow \frac{\widetilde{K}_{2}(I)}{J} \rightarrow K_{2}(I)(-2) \rightarrow 0
$$

By the long exact sequence of Tor, we know that

$$
\begin{aligned}
& S(-e-1)^{b_{e}} \quad S(-3)^{b_{2}} \quad S(-2)^{b_{1}} \\
& 0 \rightarrow \oplus S(-e-2)^{b_{e}} \rightarrow \cdots \rightarrow \oplus S(-4)^{b_{2}} \rightarrow \oplus S(-3)^{b_{1}} \rightarrow \tilde{K}_{2}(I) / J \rightarrow 0 .
\end{aligned}
$$

Similarly, we can compute the syzygy of $\widetilde{K}_{i}(I) / J$ for any $i$, and we get the desired resolution of $I / J=\widetilde{K}_{\infty}(I) / J$ as an $S$-module in the end.

Remark 2.6. For the next section, we point out some useful facts as follows:

(a) (Reduction of syzygies) From the sequence (2.2), we have an isomorphism

$$
\operatorname{Tor}_{i}^{S}(I / J)_{i+j} \simeq \operatorname{Tor}_{i}^{S}\left(\widetilde{K}_{d}(I) / J\right)_{i+j} \text { for any } d \geq j-1 \text {. }
$$

In other words, the syzygies of an infinitely generated $S$-module $I / J$ can be computed from the syzygies of a finitely generated $S$-module $\widetilde{K}_{i}(I)$. Further, if all $K_{i}(I)$ 's allow only linear syzygies at each step (i.e. $\operatorname{Tor}_{a}^{S}\left(K_{i}(I)\right)_{a+b}$ $=0 \forall a$ and $\forall b \neq i+1)$, then

$$
\operatorname{Tor}_{i}^{S}(I / J)_{i+j} \simeq \operatorname{Tor}_{i}^{S}\left(K_{j-1}(I)(-j+1)\right)_{i+j} \text { for any } i, j
$$

as Proposition 2.5 (b) shows us that the syzygies of $I / J$ essentially come just from the Koszul syzygies of $\left\{x_{0}{ }^{\alpha} \ell_{1}, \ldots, x_{0}{ }^{\alpha} \ell_{e}\right\}$ of $K_{\alpha}(I)$.

(b) (Commutativity of $x_{0}$-multiplication) Consider the $S$-module homomorphism $\phi: I \rightarrow I / J$, the natural quotient map, and also consider multiplication maps in both $I$ and $I / J$. This multiplication $\times x_{0}$ is not well-defined in $I / J$, while it is a well-defined $S$-module homomorphism in $I$. But if $X$ is quadratic and $q$ is a smooth point, then, by Proposition 2.5 (b) and (a) above, we have a commuting diagram at the Tor-level:

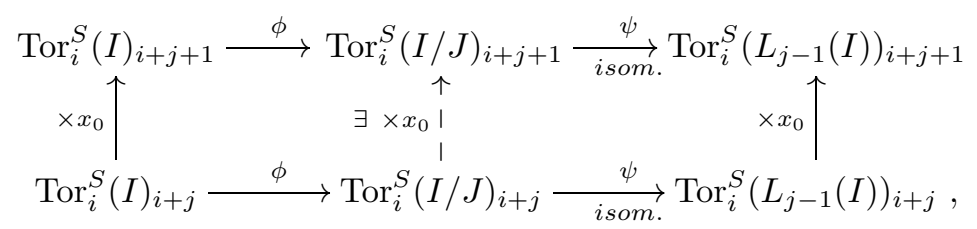

where $L_{j-1}(I):=K_{j-1}(I)(-j+1)$ and each row $\psi \circ \phi$ is induced from the $S$ homomorphism $I \rightarrow L_{j-1}(I)$ given by $f=x_{0}^{j-1} \bar{f}_{j-1}+x_{0}^{j-2} \bar{f}_{j-2}+\cdots+\bar{f}_{0} \mapsto$ $x_{0}^{j-1} \bar{f}_{j-1}$ which naturally commutes with the $x_{0}$-multiplication. 
Remark 2.7. (a) (Outer projection case) We can also consider the outer projection by a similar method. In this case $K_{i}(I)$ always stabilizes at least at the $d$-th step to $(1)=S$ if $I$ satisfies $\mathbf{N}_{d, 1}$. A more interesting fact is that $K_{d-1}(I)$ consists of linear forms with the $\mathbf{N}_{d, 2}$-condition. Especially, suppose that $X$ satisfies property $\mathbf{N}_{2,2}$ and $q=(1,0, \ldots, 0) \notin X$. Then $K_{1}(I)$ is an ideal of linear forms $I_{\Sigma}$ defining the singular locus $\Sigma$ of $\pi_{q}$ in $X_{q} \subset \mathbb{P}^{N-1}$ (see [AK11] for details). By a similar method as in the inner projection, we see that $I / J$ has simple $S$-module syzygies such that:

$$
\begin{aligned}
0 \rightarrow S(-t-1)^{b_{t}} \rightarrow \cdots \rightarrow S(-3)^{b_{2}} \rightarrow & S(-2)^{b_{1}+1} \rightarrow I / J \rightarrow 0 \\
& \oplus S(-3) \\
& \oplus S(-4) \\
& \cdots
\end{aligned}
$$

where $b_{i}=\left(\begin{array}{l}t \\ i\end{array}\right), t=\operatorname{codim}\left(\Sigma, \mathbb{P}^{N-1}\right)$. So, this resolution can be used to study the outer projection case.

(b) The stabilized ideal gives important information for projections. In the outer case of $\mathbf{N}_{2, p}(p \geq 2)$, it is shown in AK11, Park08 that the dimension of $\Sigma$ determines the number of quadric equations and the arithmetic depth of projected varieties according to moving the center of projection. In our inner projection, $K_{1}(I)$ also shows us the tangential behavior of $X$ at $q$ and $T C_{q} X$ plays an important role in our problem.

Now there arise some basic and natural questions. How are the syzygies of $J$ related to the $S$-module syzygies of $I$ and to the $R$-module syzygies of $I$ ? With the assumption for property $\mathbf{N}_{2, p}$, we may ask the following question specifically: Is $J$ generated only by quadrics if $I$ is? There might be cubic generators such as $\ell_{i} Q_{0, j}-\ell_{j} Q_{0, i}\left(=\ell_{j} \cdot\left[x_{0} \ell_{i}-Q_{0, i}\right]-\ell_{i} \cdot\left[x_{0} \ell_{j}-Q_{0, j}\right]\right)$ in $J$ (see (1.1) in Section 1). If not, how about the case of $\mathbf{N}_{2,2}$ ? What can we say about higher linear syzygies of $X_{q}$ ? We will answer these kinds of syzygy and elimination problems and derive stronger results by using the elimination mapping cone sequence and the partial elimination ideal theory in the next section.

\section{EMBEDDED LINEAR SYZYGIES AND APPLICATIONS}

Recently, D. Eisenbud et al. showed that with the assumption for some property $\mathbf{N}_{2, p}$, the syzygies of $X$ restrict surjectively to the syzygies of linear sections in their paper 'Restricting linear syzygies', EGHP05. We consider in this section the behavior of the syzygies under inner projections and we present one of our main theorems on 'embedded linear syzygies' which is the natural projection-analogue of the linear section case.

Theorem 3.1. Let $X \subset \mathbb{P}^{N}$ be a nondegenerate reduced quadratic scheme whose saturated ideal $I_{X}$ satisfies property $\boldsymbol{N}_{2, p}$ for some $p \geq 1$ and let $q \in X$ be a smooth point. Consider the inner projection $\pi_{q}: X \rightarrow X_{q} \subset \mathbb{P}^{N-1}$. Then there is an injection between the minimal free resolutions of $I_{X_{q}}$ and $I_{X}$ up to the first $(p-1)$-th step, i.e.

$$
\exists f: \operatorname{Tor}_{i}^{S}\left(I_{X_{q}}, k\right)_{i+j} \hookrightarrow \operatorname{Tor}_{i}^{R}\left(I_{X}, k\right)_{i+j} \text { for } 0 \leq i \leq p-2, \forall j \in \mathbb{Z}
$$

which is induced by the natural inclusion $I_{X_{q}} \hookrightarrow I_{X}$ and the elimination mapping cone sequence (see Theorem 2.1 (a)). 
Remark 3.2. The method used to prove Theorem 3.1 is, in fact, available for any ideal $I$ defining $X$ and $J=I \cap S$ defining $X_{q}$ scheme-theoretically.

Proof. We have a basic short exact sequence of $S$-modules,

$$
0 \longrightarrow I_{X_{q}} \longrightarrow I_{X} \longrightarrow I_{X} / I_{X_{q}} \longrightarrow 0 \text {. }
$$

From the long exact sequence of (3.1) and the mapping cone sequence of $I_{X}$ in (2.1), we have a diagram

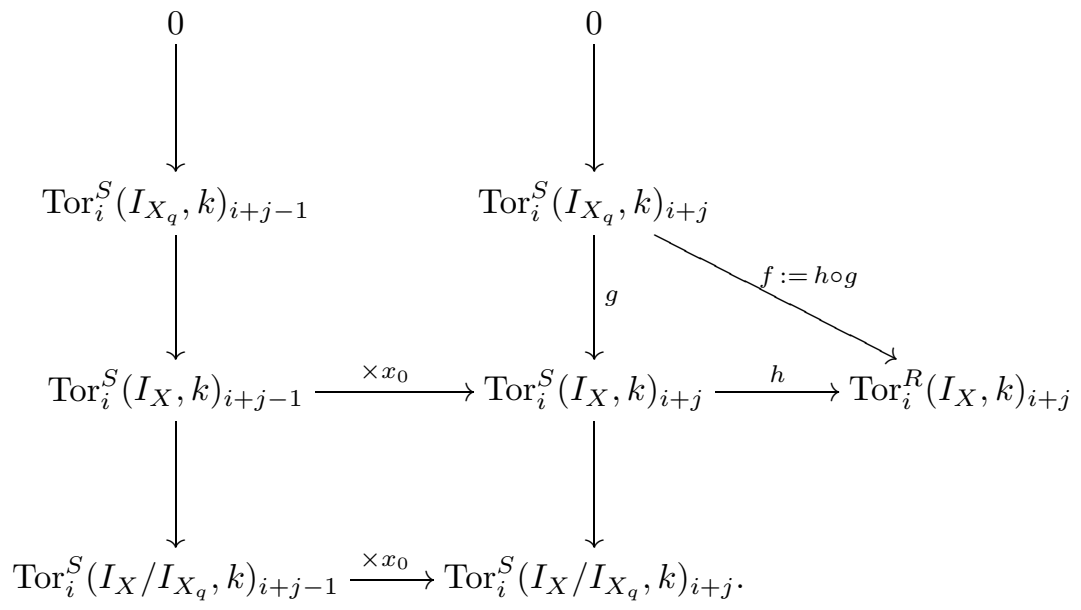

For any $0 \leq i \leq p-2$, we proceed with $j$ case by case.

Case 1. $j \leq 1$ : Since $\operatorname{Tor}_{i}^{S}\left(I_{X_{q}}, k\right)_{i+j}=0$, it is obviously injected to $\operatorname{Tor}_{i}^{R}\left(I_{X}, k\right)_{i+j}$ by $f$.

Case 2. $j=2$ (i.e. linear syzygy cases for each $i$ ): From (3.1), we have

$$
\operatorname{Tor}_{i+1}^{S}\left(I_{X} / I_{X_{q}}, k\right)_{i+2} \longrightarrow \operatorname{Tor}_{i}^{S}\left(I_{X_{q}}, k\right)_{i+2} \stackrel{g}{\longrightarrow} \operatorname{Tor}_{i}^{S}\left(I_{X}, k\right)_{i+2} .
$$

Since $q$ is a smooth point, with the $N_{2,1}$ condition we know the syzygy structures of $I_{X} / I_{X_{q}}$ as an $S$-module by Proposition 2.5 (b). This shows that

$$
\operatorname{Tor}_{i+1}^{S}\left(I_{X} / I_{X_{q}}, k\right)_{i+2}=0
$$

implying that $g$ is injective. Since $X$ is nondegenerate, $\operatorname{Tor}_{i}^{S}\left(I_{X}, k\right)_{i+1}=0$ and $h$ is also injective at the horizontal mapping cone sequence of the above diagram. Hence $f$ is injective in this case, too.

Case 3. $j \geq 3$ : First note that $\operatorname{Tor}_{i}^{R}\left(I_{X}, k\right)_{i+j}=0$ for $0 \leq i \leq p-1, j \geq 3$ by the assumption of property $\mathbf{N}_{2, p}$. We will show that $g$ is injective and $\operatorname{Tor}_{i}^{S}\left(I_{X}, k\right)_{i+j}$ is isomorphic to $\operatorname{Tor}_{i}^{S}\left(I_{X} / I_{X_{q}}, k\right)_{i+j}$ for $0 \leq i \leq p-2$. Then we can conclude that $\operatorname{Tor}_{i}^{S}\left(I_{X_{q}}, k\right)_{i+j}=0$, so $f$ is injective for $0 \leq i \leq p-2$.

Consider the commutative diagram (by Remark 2.6) in the third quadrant part of the above diagram. Repeating this diagram by multiplying $x_{0}$ sufficiently, we 
have the following diagram:

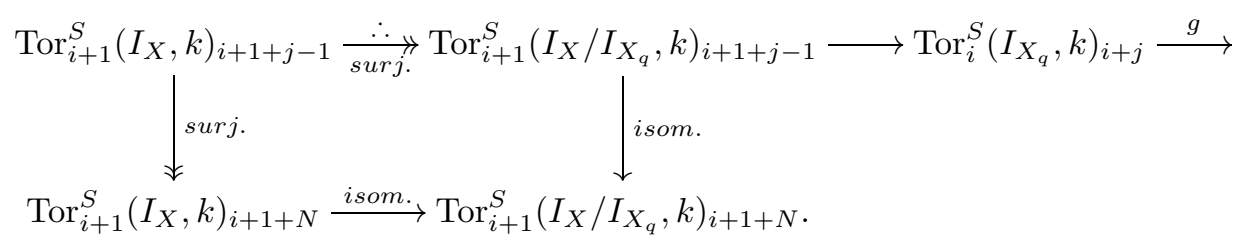

The left vertical map is surjective from Theorem 2.1(b), and the right one is an isomorphism by the syzygy structures of $I_{X} / I_{X_{q}}$ in Proposition 2.5 (b). Since $I_{X_{q}}$ is a finite $S$-module, $\operatorname{Tor}_{i}^{S}\left(I_{X_{q}}, k\right)_{i+N}=0$ for sufficiently large $N$, so we get the below (second row) isomorphism. Therefore the map

$$
\operatorname{Tor}_{i+1}^{S}\left(I_{X}, k\right)_{i+1+j-1} \rightarrow \operatorname{Tor}_{i+1}^{S}\left(I_{X} / I_{X_{q}}, k\right)_{i+1+j-1}
$$

is surjective, and $g$ is injective.

Similarly, we can have the desired isomorphism between $\operatorname{Tor}_{i}^{S}\left(I_{X}, k\right)_{i+j}$ and $\operatorname{Tor}_{i}^{S}\left(I_{X} / I_{X_{q}}, k\right)_{i+j}$ as follows:

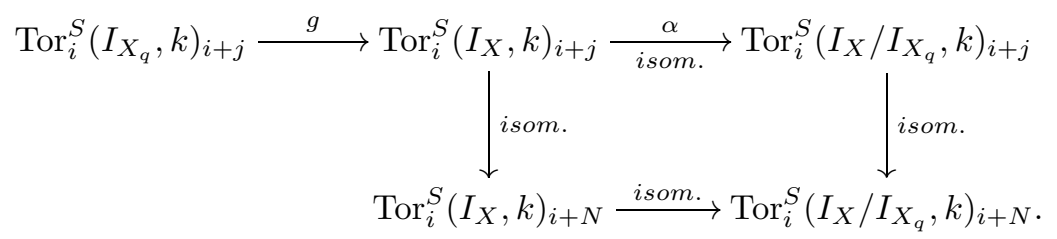

In this case, the mapping cone construction gives the left vertical isomorphism by Theorem 2.1 (b). So the above map $\alpha$ is an isomorphism as we wish, and $\operatorname{Tor}_{i}^{S}\left(I_{X_{q}}, k\right)_{i+j}=0$ for $0 \leq i \leq p-2, j \geq 3$.

This main Theorem 3.1 tells us that all the $S$-module syzygies of $X_{q}$ are exactly the very ones which are already embedded in the linear syzygies of $X$ as an $R$ module. This doesn't hold for outer projection and inner projection of varieties with $\mathbf{N}_{d, p}(d \geq 3)$.

Example 3.3. Let $C$ be a rational normal curve in $\mathbb{P}^{3}$ and $I_{C}$ be the homogeneous ideal $\left(x_{0} x_{2}-x_{1}^{2}, x_{0} x_{1}-x_{1} x_{3}-x_{2}^{2}, x_{0}^{2}-x_{0} x_{3}-x_{1} x_{2}\right)$ under suitable coordinate change. We know that $C$ is 2-regular and consider an outer projection of $C$ from $q=(1,0,0,0)$. Then $I_{C_{q}}=\left(x_{1}^{3}-x_{1} x_{2} x_{3}-x_{2}^{3}\right)$ has a cubic generator (i.e. $\left.\mathbf{N}_{3,1}\right)$. Since $x_{1}^{3}-x_{1} x_{2} x_{3}-x_{2}^{3}=\left(-x_{1}\right) \cdot\left[x_{0} x_{2}-x_{1}^{2}\right]+x_{2} \cdot\left[x_{0} x_{1}-x_{1} x_{3}-x_{2}^{2}\right]$, this is zero in $\operatorname{Tor}_{0}^{R}\left(I_{C}\right)_{3}$ and $\operatorname{Tor}_{0}^{S}\left(I_{C_{q}}\right)_{3} \rightarrow \operatorname{Tor}_{0}^{R}\left(I_{C}\right)_{3}$ is not injective. In general, if we take the center $q \in L \simeq \mathbb{P}^{2}$ which is a multisecant (e.g. at least a 4-secant) 2-plane, then for outer and inner projection cases there is a multisecant line to $X_{q}$. So, the defining equations of $X_{q}$ may have larger degrees.

As an immediate consequence, we have the following corollary.

Corollary 3.4 (Property $\mathbf{N}_{2, p-1}$ of inner projections). Let $X \subset \mathbb{P}^{N}$ be a nondegenerate reduced quadratic scheme satisfying property $\boldsymbol{N}_{2, p}$ for some $p \geq 2$ and let $q \in X$ be a smooth point. Then, the inner projection $X_{q}$ is also quadratic and satisfies property $\boldsymbol{N}_{2, p-1}$.

Proof. Case $3, j \geq 3$ in the proof of Theorem 3.1 is a proof of this corollary. 
Remark 3.5. Here are some remarks on Corollary 3.4.

(a) This corollary can be easily extended to the case of a general linear subspace $\Lambda \simeq \mathbb{P}^{t}$ such that $\operatorname{dim} X \cap \Lambda$ is zero. Precisely, if $\Lambda$ does not meet $\operatorname{Sing}(X)$ and $\langle\Lambda \cap X\rangle=\Lambda$, then the $t+1$ points of $\Lambda \cap X$ are in linearly general position so that $X_{\Lambda}$ satisfies $\mathbf{N}_{2, p-t-1}$ by successive inner projections. To complete this question in Problem (a) for any linear subspace $\Lambda$, it remains to consider how the projections from a singular center or a linear subvariety contained in $X$ behave (see Questions $5.3,5.5$ ).

(b) For a smooth irreducible variety $X \subset \mathbb{P}\left(H^{0}(\mathcal{L})\right)$ with the condition $\mathbf{N}_{p}$ $(p \geq 1)$ embedded by the complete linear system of a very ample line bundle $\mathcal{L}$ on $X$, Y. Choi, P. Kang and S. Kwak showed that the inner projection $X_{q}$ is smooth and satisfies property $\mathbf{N}_{p-1}$ for any $q \in X \backslash \operatorname{Trisec}(X)$; i.e. property $\mathbf{N}_{p-1}$ holds for $\left(\operatorname{Bl}_{q}(X), \sigma^{*} \mathcal{L}-E\right)$ by using vector bundle techniques and Koszul cohomology methods due to Green-Lazarsfeld (see [CKK06]). Our Corollary 3.4 extends this result to the category of reduced projective schemes satisfying property $\mathbf{N}_{2, p}$ with any smooth point $q \in X$. Note that this uniform behavior looks unusual in the sense that linear syzygies of outer projections heavily depend on moving the center of projection in an ambient space $\mathbb{P}^{N}$ (see CKP08, KP05, Park08]).

In order to understand the Betti table of inner projections, we need to consider defining equations of inner projections, depth, and the Castelnuovo-Mumford regularity.

Proposition 3.6 (Quadratic equations of inner projections). Let $X \subset \mathbb{P}^{N}$ be $a$ nondegenerate reduced scheme with a defining ideal $I$ and any (possibly singular) point $q \in X$. For the inner projection $X_{q} \subset \mathbb{P}^{N-1}$, we have

(a) $\beta_{0,2}(J)=\beta_{0,2}(I)-\beta_{0,1}\left(K_{1}(I)\right)$, where $J$ is the $x_{0}$-elimination ideal of $I$ as usual.

(b) Furthermore, if $I$ is quadratic (so $X$ is quadratic), then we have $\beta_{0,2}(J)=$ $\beta_{0,2}(I)-N+\operatorname{dim} T_{q} X$. In particular, in the case of $I=I_{X}$ it coincides with

$$
h^{0}\left(\mathbb{P}^{N-1}, \mathcal{J}_{X_{q}}(2)\right)=h^{0}\left(\mathbb{P}^{N}, \mathcal{J}_{X}(2)\right)-N+\operatorname{dim} T_{q} X .
$$

Proof. As in the proof of Theorem 3.1, there is a long exact sequence such that

$$
\rightarrow \operatorname{Tor}_{1}^{S}(I / J, k)_{2} \rightarrow \operatorname{Tor}_{0}^{S}(J, k)_{2} \rightarrow \operatorname{Tor}_{0}^{S}(I, k)_{2} \rightarrow \operatorname{Tor}_{0}^{S}(I / J, k)_{2} \rightarrow 0 .
$$

From the reduction of syzygies (2.3) in Remark 2.6, we have

$$
\left\{\begin{array}{l}
\operatorname{Tor}_{1}^{S}(I / J, k)_{2} \simeq \operatorname{Tor}_{1}^{S}\left(K_{1}(I)(-1), k\right)_{2}=\operatorname{Tor}_{1}^{S}\left(K_{1}(I), k\right)_{1}=0, \\
\operatorname{Tor}_{0}^{S}(I / J, k)_{2} \simeq \operatorname{Tor}_{0}^{S}\left(K_{1}(I)(-1), k\right)_{2}=\operatorname{Tor}_{0}^{S}\left(K_{1}(I), k\right)_{1},
\end{array}\right.
$$

which implies the desired formula in (a) directly.

If $I$ is a quadratic ideal, then by Proposition 2.5, the $K_{i}(I)$ stabilizes at $i=1$ and $K_{1}(I)$ defines the tangent cone $T C_{q} X$. We may write

$$
K_{1}(I)=\left(\ell_{1}, \ldots, \ell_{t} \text {, higher degree polynomials }\right),
$$

where $I=\left(x_{0} \ell_{1}-Q_{0,1}, \ldots, x_{0} \ell_{t}-Q_{0, t}, Q_{1}, \ldots, Q_{s}\right)$ just as our convention. Among the elements of $K_{1}(I)$ the generators of $K_{1}(I)_{1},\left\{\ell_{1}, \ldots, \ell_{t}\right\}$ define the tangent space $T_{q} X$ so that we have $\beta_{0,1}\left(K_{1}(I)\right)=\operatorname{codim}\left(T_{q} X, \mathbb{P}^{N}\right)$. 
Remark 3.7. In the outer projection case, there is a formula $h^{0}\left(\mathcal{J}_{X_{q}}(2)\right)=h^{0}\left(\mathcal{J}_{X}(2)\right)$ $-\left(N-\operatorname{dim} \Sigma_{q}(X)\right)$ if $X$ satisfies property $\mathbf{N}_{2,2}$ (see proposition 4.11 in [AK11, theorem 3.3 in [Park08]). This also shows that there is a tendency of having more quadrics for projected varieties as $q$ is getting closer to $X$. Note that the negative value of $h^{0}\left(\mathcal{J}_{X_{q}}(2)\right)$ implies that there is no quadric vanishing on $X_{q}$. By this fact, we can expect that the inner projection case has more linear syzygies as Corollary 3.4 shows.

The next question is how many quadrics defining $X$ are required to satisfy property $\mathbf{N}_{2, p}$, and we give the sharp lower bound in the following.

Corollary 3.8 (Neccesary lower bound for property $\mathbf{N}_{2, p}$ ). Let $X$ be a nondegenerate reduced quadratic scheme in $\mathbb{P}^{r+e}$ of codimension $e$ and $I$ be the quadratic ideal of $X$. Suppose that I satisfies property $\boldsymbol{N}_{2, p}$ and $\beta_{0,2}(I)$ is the number of generators of $I$. Then $\beta_{0,2}(I)$ is not less than $L B_{p}$ as follows:

$$
L B_{p}=e \cdot p-\frac{p(p-1)}{2} \leq \beta_{0,2}(I) \leq \beta_{0,2}\left(I_{X}\right)\left(=h^{0}\left(\mathcal{J}_{X}(2)\right)\right) .
$$

Proof. Let's take a smooth point $q_{0}$ in $X$ and project $X$ from $q_{0}$. Let $X^{(1)}$ be the image (the Zariski closure) and $I^{(1)}$ be the elimination ideal of $I$. Then, from Proposition 3.6 we get

$$
\beta_{0,2}\left(I^{(1)}\right)=\beta_{0,2}(I)-(r+e)+r .
$$

We also know that $I^{(1)}$ defines $X^{(1)}$ scheme-theoretically and satisfies property $\mathbf{N}_{2, p-1}$. Take another smooth point $q_{1}$ in $X^{(1)}$ and project it from $q_{1}$. Then, with the same notation, we have

$$
\beta_{0,2}\left(I^{(2)}\right)=\beta_{0,2}\left(I^{(1)}\right)-(r+e-1)+r .
$$

Taking successive inner projections, we get

$$
\beta_{0,2}\left(I^{(p-1)}\right)=\beta_{0,2}\left(I^{(p-2)}\right)-(r+e-p+2)+r .
$$

Summing up both sides of the above equations, this gives

$$
\beta_{0,2}\left(I^{(p-1)}\right)=\beta_{0,2}(I)-\frac{(p-1)(2 r+2 e-p+2)}{2}+r(p-1),
$$

and we know that $X^{(p-1)}$ is still cut by quadrics (i.e. $\left.\mathbf{N}_{2,1}\right)$. So $\beta_{0,2}\left(I^{(p-1)}\right)$ is not less than $\operatorname{codim} X^{(p-1)}=(r+e)-p+1-r=e-p+1$. If we plug in this inequality to $(*)$, we get the desired bound $\mathrm{LB}_{p}$.

Remark 3.9. This bound is sharp for $p=1$ by complete intersections, $p=e-1$ by del Pezzo varieties (see Theorem 4.3 (b)), and $p=e$ by minimal degree varieties. Note also that the upper bound for $\beta_{0,2}\left(I_{X}\right)$ for a nondegenerate integral subscheme $X \subset \mathbb{P}^{r+e}$ of codimension $e$ is $\frac{e(e+1)}{2}$ and this maximum number can be attained if and only if the variety $X$ is of minimal degree from Corollaries 5.4,5.8 in [ak99].

Remark 3.10 (Degree bound by property $\mathbf{N}_{2, p}$ ). Recently, A. Alzati and J.C. Sierra obtained a bound of quadrics for $\mathbf{N}_{2,2}$ by paying attention to the structures of the rational map associated to the linear system of quadrics defining $X$, which coincides with our bound $\mathrm{LB}_{2}$ (see AS10]). They also derive a degree bound in terms of codimension $e,\left(\begin{array}{l}d \\ 2\end{array}\right) \leq\left(\begin{array}{c}2 e-1 \\ e-1\end{array}\right)$ whose asymptotic behavior is $2^{e} / \sqrt[4]{\pi e}$ and describe the equality condition: this holds if and only if the equality of $\mathrm{LB}_{2}$ holds. From this theorem, in the case of $p=2$ we also have a rigid condition on the degree of 
the boundary $X$ as if we get some rigidity when $p=1, e-1$, and $e$ (see Remark 3.9 and Theorem 4.3). Using our inner projection method (e.g. Corollary 3.8), we could improve this degree bound a little as follows:

$\left(\begin{array}{c}d+2-p \\ 2\end{array}\right) \leq\left(\begin{array}{c}2 e+3-2 p \\ e+1-p\end{array}\right), \quad d \sim 2^{e+2-p} / \sqrt[4]{\pi e} \quad$ (as e becomes sufficiently large)

under the assumption of property $\mathbf{N}_{2, p}(p \geq 2)$ of $X$.

Example 3.11. It would be interesting to know that if $e \leq \beta_{0,2}\left(I_{X}\right)<2 e-1$, then $X$ has always at least a syzygy of defining equations which is not linear because property $\mathbf{N}_{2,2}$ does not hold for $X$. For example, let $C$ be the general embedding of degree 19 in $\mathbb{P}^{7}$ of genus 12 . Then $C$ is a smooth arithmetically Cohen-Macaulay curve which is cut out scheme-theoretically by 9 quadrics, but the homogeneous ideal $I_{C}$ is generated by 9 quadrics and 2 cubics (see Katz93 for details). These quadratic generators should have at least a syzygy of higher degree as well as linear syzygies.

Example 3.12 (Veronese embedding $v_{d}\left(\mathbb{P}^{n}\right)$ ). It is shown that $v_{d}\left(\mathbb{P}^{n}\right)$ fails property $\mathbf{N}_{2,3 d-2}$ for $n \geq 2, d \geq 3$ and conjectured that $v_{d}\left(\mathbb{P}^{n}\right)$ satisfies property $\mathbf{N}_{2,3 d-3}$ for $n \geq 2, d \geq 3$ (see [OP01, EGHP05]). We can also verify the failure of property $\mathbf{N}_{2, p}$ of the Veronese embedding $X=v_{d}\left(\mathbb{P}^{n}\right)$ for some cases by using this low bound $\mathrm{LB}_{p}$. For example, when $n=2, d=3, p=3 d-2=7$, we get $\beta_{0,2}\left(I_{X}\right)=27$ and $\mathrm{LB}_{7}=7 \cdot 7-\left(\begin{array}{l}7 \\ 2\end{array}\right)=28$. Therefore, $v_{3}\left(\mathbb{P}^{2}\right)$ fails to satisfy $\mathbf{N}_{2,7}$. Similarly, $v_{2}\left(\mathbb{P}^{3}\right)$ fails property $\mathbf{N}_{2,6}$. However, it does not give the reason why $v_{3}\left(\mathbb{P}^{3}\right)$ does fail to be $\mathbf{N}_{2,7}$ for the case $n=d=3, p=3 d-2=7, e=16$, because $\beta_{0,2}\left(I_{X}\right)=126>91=\mathrm{LB}_{7}$. For such a $p$ in the middle area of $1 \leq p \leq e, \mathrm{LB}_{p}$ seems not to give quite sufficient information for property $\mathbf{N}_{2, p}$, while it may be more effective to decide $\mathbf{N}_{2, p}$ of a given variety for rather large $p$ among $1 \leq p \leq e$.

\section{Arithmetic DePth And SYZYGetic Rigidity}

Now, we proceed to investigate the depth of inner projections to understand the shape of the Betti table and Castelnuovo-Mumford regularity. In this section we always consider the saturated ideal $I_{X}$ among ideals defining $X$ because $\operatorname{depth}_{R}(R / I)=0$ for any defining ideal $I$ which is not saturated. The following result looks very surprising when we compare this with the outer projection case (see Remark 4.2).

Theorem 4.1 (The depth of inner projections). Let $X \subset \mathbb{P}^{N}$ be a nondegenerate reduced subscheme and $I_{X}$ be generated by quadrics. Consider the inner projection $\pi_{q}: X \rightarrow X_{q} \subset \mathbb{P}^{N-1}$ from a smooth point $q \in X$. Then,

(a) (the projective dimension of $\left.S / I_{X_{q}}\right) \operatorname{pd}_{S}\left(S / I_{X_{q}}\right)=\operatorname{pd}_{R}\left(R / I_{X}\right)-1$;

(b) (the arithmetic depth of $X_{q}$ ) $\operatorname{depth}_{R}(X)=\operatorname{depth}_{S}\left(X_{q}\right)$. In particular, $X$ is arithmetically Cohen-Macaulay if and only if $X_{q}$ is also.

Proof. (a) We know $\operatorname{pd}_{R}\left(R / I_{X}\right) \geq e=\operatorname{codim} X$. Let $l$ be $\operatorname{pd}_{R}\left(R / I_{X}\right)($ so, $l \geq e)$, and $j_{0}=\max \left\{j \mid \operatorname{Tor}_{l}^{R}\left(R / I_{X}\right)_{l+j} \neq 0\right\}$. 
Case 1) Non-Cohen-Macaulay case (i.e. $l=e+\alpha, \alpha \geq 1$ ). First of all, we have the following diagram from the exact sequence (3.1):

\begin{tabular}{|c|c|c|c|c|c|}
\hline$i$ of $\operatorname{Tor}_{i}^{S}\left(S / I_{X_{q}}\right)$ & $0 \rightarrow$ & $\begin{array}{c}I_{X_{q}} \\
\uparrow\end{array}$ & $\rightarrow$ & $\underset{\uparrow}{I_{X}} \rightarrow$ & $I_{X} / I_{X_{q}} \rightarrow 0$ \\
\hline 1 & & $\square$ & & $\square$ & $S(-2)^{e} \oplus S(-3)^{e} \oplus \cdots$ \\
\hline 2 & & $\vdots$ & & $\vdots$ & $\vdots$ \\
\hline & & $\uparrow$ & & $\uparrow$ & $\uparrow$ \\
\hline$e$ & & $\square$ & & $\square$ & $S(-e-1) \oplus S(-e-2) \oplus \cdots$ \\
\hline & & $\uparrow$ & & $\uparrow$ & $\uparrow$ \\
\hline$e+1$ & & $\square$ & $\cong$ & $\square$ & 0 \\
\hline$\vdots$ & & $\vdots$ & $\vdots$ & $\vdots$ & $\vdots$ \\
\hline$l=e+\alpha$ & & $\square$ & $\cong$ & $\boldsymbol{\square}$ & 0 \\
\hline
\end{tabular}

From this diagram, we get $\operatorname{Tor}_{l}^{S}\left(R / I_{X}\right) \cong \operatorname{Tor}_{l}^{S}\left(S / I_{X_{q}}\right)$ as finite $k$-vector spaces. Since $\operatorname{Tor}_{l+1}^{R}\left(R / I_{X}\right)_{l+1+j}=0$ for all $j\left(\because \operatorname{pd}_{R}\left(R / I_{X}\right)=l\right)$ and $\operatorname{Tor}_{l}^{R}\left(R / I_{X}\right)_{l+j}=0$ for $j>j_{0}$, we can observe using the mapping cone sequence (2.1) that

$$
\ldots \stackrel{\times x_{0}}{\longrightarrow} \operatorname{Tor}_{l}^{S}\left(R / I_{X}\right)_{l+j} \stackrel{\times x_{0}}{\longrightarrow} \cdots \stackrel{\times x_{0}}{\longrightarrow} \operatorname{Tor}_{l}^{S}\left(R / I_{X}\right)_{l+j_{0}} \stackrel{\times x_{0}}{\rightarrow} \cdots .
$$

So we have $\operatorname{Tor}_{l}^{S}\left(R / I_{X}\right)=0$, because it is finite dimensional. This means that $\operatorname{Tor}_{l}^{S}\left(S / I_{X_{q}}\right) \cong \operatorname{Tor}_{l}^{S}\left(R / I_{X}\right)=0$.

Next, we claim that $\operatorname{Tor}_{l-1}^{S}\left(S / I_{X_{q}}\right) \neq 0$, which implies that $\operatorname{pd}_{S}\left(S / I_{X_{q}}\right)=l-1$. If $\alpha \geq 2$, then we have $\operatorname{Tor}_{l-1}^{S}\left(S / I_{X_{q}}\right) \simeq \operatorname{Tor}_{l-1}^{S}\left(R / I_{X}\right)$. Since $\operatorname{Tor}_{l}^{S}\left(R / I_{X}\right)=0$, we have a nontrivial kernel of the $\times x_{0}$ map in $I_{X}$ from the mapping cone sequence (2.1)

$$
\begin{array}{ccc}
(*) 0 \rightarrow \operatorname{Tor}_{l}^{R}\left(R / I_{X}\right)_{l+j_{0}} \hookrightarrow & \operatorname{Tor}_{l-1}^{S}\left(R / I_{X}\right)_{l-1+j_{0}} \stackrel{\times x_{0}}{\rightarrow} & \operatorname{Tor}_{l-1}^{S}\left(R / I_{X}\right)_{l-1+j_{0}+1} \cdots \\
\hdashline & \operatorname{Tor}_{l-2}^{S}\left(I_{X}\right)_{l-1+j_{0}} & \operatorname{Tor}_{l-2}^{S}\left(I_{X}\right)_{l-1+j_{0}+1} .
\end{array}
$$

This implies that $\operatorname{Tor}_{l-1}^{S}\left(R / I_{X}\right) \simeq \operatorname{Tor}_{l-1}^{S}\left(S / I_{X_{q}}\right) \neq 0$ as wished. So, let us focus on the case $\alpha=1$ and so, $l=e+1$. Consider the following sequence and commutative diagram:

$$
\begin{gathered}
\operatorname{Tor}_{l-1}^{S}\left(I_{X} / I_{X_{q}}\right)=0 \rightarrow \operatorname{Tor}_{l-2}^{S}\left(I_{X_{q}}\right) \rightarrow \operatorname{Tor}_{l-2}^{S}\left(I_{X}\right) \rightarrow \operatorname{Tor}_{l-2}^{S}\left(I_{X} / I_{X_{q}}\right) \rightarrow \cdots \\
\operatorname{Tor}_{l-2}^{S}\left(I_{X}\right)_{e+j_{0}} \simeq S\left(-e-j_{0}\right)^{\square} \otimes k \stackrel{f_{e+j_{0}}}{\rightarrow}
\end{gathered}
$$

where $h, g$ are induced by the multiplication of $x_{0}^{n}$ and $g$ is an isomorphism. To check that $\operatorname{Tor}_{l-1}^{S}\left(S / I_{X_{q}}\right) \cong \operatorname{Tor}_{l-2}^{S}\left(I_{X_{q}}\right) \neq 0$, it is enough to show that $f: \operatorname{Tor}_{l-2}^{S}\left(I_{X}\right) \rightarrow$ $\operatorname{Tor}_{l-2}^{S}\left(I_{X} / I_{X_{q}}\right)$ is not injective because $\operatorname{Tor}_{l-1}^{S}\left(I_{X} / I_{X_{q}}\right)=0$. Now let me explain why $f$ is not injective. We get the isomorphism map below for $c \gg 0$ because $\operatorname{Tor}_{i}^{S}\left(I_{X_{q}}\right)$ are finite-dimensional graded vector spaces and also $h$ is not injective by $(*)$. From Remark (2.6), this diagram commutes and $f_{e+j_{0}}$ has a nontrivial kernel. Hence $f: \operatorname{Tor}_{l-2}^{S}\left(I_{X}\right) \rightarrow \operatorname{Tor}_{l-2}^{S}\left(I_{X} / I_{X_{q}}\right)$ is not injective and $\operatorname{Tor}_{l-1}^{S}\left(S / I_{X_{q}}\right)_{e+j_{0}}=$ $\operatorname{Tor}_{l-2}^{S}\left(I_{X_{q}}\right)_{e+j_{0}} \neq 0$. 
Case 2) Cohen-Macaulay case (i.e. $l=e, \alpha=0$ ). In this case, we have the long exact sequence on Tor as follows:

$$
0=\operatorname{Tor}_{e}^{S}\left(I_{X} / I_{X_{q}}\right) \rightarrow \operatorname{Tor}_{e-1}^{S}\left(I_{X_{q}}\right) \rightarrow \operatorname{Tor}_{e-1}^{S}\left(I_{X}\right) \stackrel{f}{\longrightarrow} \begin{gathered}
S(-e-1) \oplus \\
S(-e-2) \oplus \\
\ldots
\end{gathered}
$$

Since $\operatorname{pd}_{R}\left(R / I_{X}\right)=e, \operatorname{Tor}_{e+1}^{R}\left(R / I_{X}\right)=0$ and we have an injection

$$
\operatorname{Tor}_{e-1}^{S}\left(I_{X}\right)_{e+j}=\operatorname{Tor}_{e}^{S}\left(R / I_{X}\right)_{e+j} \stackrel{\times x_{0}^{n}}{\longrightarrow} \operatorname{Tor}_{e}^{S}\left(R / I_{X}\right)_{e+j+n}=\operatorname{Tor}_{e-1}^{S}\left(I_{X}\right)_{e+j+n}
$$

for any $j, n$ from the mapping cone sequence (2.1). By almost the same argument using the commuting diagram as in Case 1), $\alpha=1$, we can conclude that $f$ : $\operatorname{Tor}_{e-1}^{S}\left(I_{X}\right) \rightarrow \operatorname{Tor}_{e-1}^{S}\left(I_{X} / I_{X_{q}}\right)$ is injective and $\operatorname{Tor}_{e}^{S}\left(S / I_{X_{q}}\right)=0$. So, this means that $\operatorname{pd}_{S}\left(S / I_{X_{q}}\right) \leq e-1$. But we know that $\operatorname{pd}_{S}\left(S / I_{X_{q}}\right) \geq \operatorname{codim}\left(X_{q}\right)=e-1$; therefore $\operatorname{pd}_{S}\left(S / I_{X_{q}}\right)=e-1$. On the other hand, (a) implies that $\operatorname{depth}_{R}(X)=$ $\operatorname{depth}_{S}\left(X_{q}\right)$ by the Auslander-Buchsbaum formula.

Remark 4.2. Let $X \subset \mathbb{P}^{n}$ be a reduced scheme satisfying property $\mathbf{N}_{2, p}(p \geq 2)$. Let $\Sigma_{q}(X)=\left\{x \in X \mid \pi_{q}{ }^{-1}\left(\pi_{q}(x)\right)\right.$ has length $\left.\geq 2\right\}$ be the secant locus of the outer projection. We would like to remark that $\operatorname{depth}\left(X_{q}\right)=\min \left\{\operatorname{depth}(X), \operatorname{dim} \Sigma_{q}(X)+\right.$ $2\}$ for a smooth scheme $X$ (see [AK11, Park08]). On the other hand, it would be interesting to ask the following question: Is there an example such that $\operatorname{depth}\left(X_{q}\right) \neq$ $\operatorname{depth}(X)$ for inner projections?

As an interesting application of the above results, we can also prove the following rigidity theorem for the extremal (i.e. $p=e$ ) and next-to-extremal (i.e. $p=e-1)$ cases of property $\mathbf{N}_{2, p}$ of the varieties by using Corollary [3.4, Proposition 3.6] and Theorem 4.1

Theorem 4.3 (Syzygetic rigidity on property $\mathbf{N}_{2, p}$ ). Let $X$ be a nondegenerate $r$-dimensional variety (i.e. irreducible, reduced) in $\mathbb{P}^{r+e}, e=\operatorname{codim}\left(X, \mathbb{P}^{r+e}\right)$.

(a) (extremal case) $X$ satisfies property $\boldsymbol{N}_{2, e}$ if and only if $X$ is a minimal degree variety, i.e. a whole linear space $\mathbb{P}^{r+e}$, a quadric hypersurface, a cone of the Veronese surface in $\mathbb{P}^{5}$, or rational normal scrolls;

(b) (next-to-extremal case) $X$ fails property $\boldsymbol{N}_{2, e}$ but satisfies $\boldsymbol{N}_{2, e-1}$ if and only if $X$ is a del Pezzo variety, i.e. $X$ is arithmetically Cohen-Macaulay (abbr. ACM) and is of next-to-minimal degree.

Proof. Let $\delta(X):=\operatorname{deg}(X)-\operatorname{codim}(X)$ for any subvariety $X \subset \mathbb{P}^{r+e}$. Note that $\delta\left(X_{q}\right)=\delta(X)$ under an inner projection from a smooth point $q \in X$. Take successive inner projections from smooth points. Call the images (Zariski closure) $X=X^{(0)}, X^{(1)}, \ldots, X^{(e-2)}$ and $I^{(i)}$ for the elimination ideal of $I^{(i-1)}$ cutting out $X^{(i)}$ scheme-theoretically. By Corollary 3.4 we know that this $X^{(e-2)}$ has codim 2 and has property $\mathbf{N}_{2,2}$ for (a) (and $\mathbf{N}_{2,1}$ for (b), respectively). Because $X^{(e-1)}$ is a hypersurface, by Proposition 3.6 the possible $\beta_{0,2}\left(I^{(e-2)}\right)=2$ or 3 . For the case (a), take an inner projection once more and then $X^{(e-1)}$ still satisfies property $\mathbf{N}_{2,1}$, i.e., an (irreducible) quadric hypersurface. So,

$$
\beta_{0,2}\left(I^{(e-1)}\right)=1, \beta_{0,2}\left(I^{(e-2)}\right)=1+2=3 \text { and } \delta(X)=\delta\left(X^{(e-1)}\right)=1 .
$$

That is, $X$ is of minimal degree. In the case of (b), $X^{(e-2)}$ is a complete intersection of two quadrics in $\mathbb{P}^{r+2}$ and $X^{(e-1)}$ should be a cubic hypersurface. 
In particular, the projective dimension of $X^{(e-2)}$ is equal to $2=\operatorname{pd}_{R}\left(R / I_{X}\right)-$ $(e-2)$ by Theorem 4.1. Therefore,

$$
\beta_{0,2}\left(I^{(e-1)}\right)=0, \beta_{0,2}\left(I^{(e-2)}\right)=2, \delta(X)=\delta\left(X^{(e-1)}\right)=2 \text { and } \operatorname{pd}_{R}\left(R / I_{X}\right)=e,
$$

which means that $X$ is arithmetically Cohen-Macaulay and of next-to-minimal degree with $H^{0}\left(\mathcal{J}_{X}(2)\right)=\frac{(e+2)(e-1)}{2}$. By the well-known classification of varieties of next-to-minimal degree, $X$ is a del Pezzo variety. On the other hand, the curve section $C$ of a del Pezzo variety $X$ is either an elliptic normal curve or a projection of a rational normal curve from a point in $\operatorname{Sec}(C) \backslash C$. Since $X$ and $C$ have the same Betti table, $X$ satisfies property $\mathbf{N}_{2, e-1}$ but fails property $\mathbf{N}_{2, e}$.

Remark 4.4. Here are some remarks on Theorem 4.3

(a) For the smooth projectively normal variety $X, M$. Green's $\mathcal{K}_{p, 1}$-theorem in Gre84 gives a necessary condition on Theorem 4.3 (b) (i.e. $X$ is either a variety of next-to-minimal degree or a divisor of a minimal degree variety). Using our Corollary 3.4 and Depth Theorem 4.1, we could obtain the results on both $\operatorname{deg}(X)=\operatorname{codim} X+2$ and the ACM property and show the rigidity on the next-to-extremal case for any (not necessarily projectively normal) variety $X$.

(b) Classically, normal del Pezzo varieties were classified by T. Fujita in Fuj90, and every nonnormal del Pezzo variety $X$ (see BS07, Fuj90]) comes from outer projection of a minimal degree variety $\widetilde{X}$ from a point $q$ in $\operatorname{Sec}(\widetilde{X}) \backslash$ $\widetilde{X}$ satisfying $\operatorname{dim} \Sigma_{q}(\widetilde{X})=\operatorname{dim} \widetilde{X}-1$ (see Remark 4.2). Note that the dimension of the secant locus $\Sigma_{q}(\tilde{X})$ varies as $q$ moves in $\operatorname{Sec}(\widetilde{X}) \backslash \tilde{X}$. Thus one can try to classify the nonnormal del Pezzo varieties by the type of the secant loci $\Sigma_{q}(\widetilde{X})$. This is recently classified in BP10] such that there are only 8 types of nonnormal del Pezzo varieties which are not cones. For example, we find projections of a smooth cubic surface scroll $S(1,2)$ in $\mathbb{P}^{4}$ from any $q \in \mathbb{P}^{4} \backslash S(1,2)$ or projections of a smooth 3 -fold scroll $S(1,1, c)$ in $\mathbb{P}^{c+4}$ with $c>1$ from any $q \in\langle S(1,1)\rangle \backslash S(1,1, c)$, etc.

Furthermore, let's consider the following category (we say that an algebraic set $X=\bigcup X_{i}$ is connected in codimesion 1 if all the component $X_{i}$ 's can be arranged in such a way that every $X_{i} \cap X_{i+1}$ is of codimension 1 in $X$ ):

(*) \{equidimensional, connected in codimension 1 , reduced subschemes in $\mathbb{P}^{r+e}$ \}.

In the category $(*)$, we have natural notions of $\operatorname{dim} X$ and $\operatorname{deg}(X)$, which is the sum of degrees of $X_{i}$ 's. As in the category of varieties, we also have the 'basic' inequality of degree, i.e. $\operatorname{deg}(X) \geq \operatorname{codim} X+1$, so it is worthwhile to think of 'minimal degree' or 'next-to-minimal degree' in this category.

Using the same methods, Theorem 4.3 can be easily extended for this category. We call $X$ (or the sequence) linearly joined whenever all the components can be ordered $X_{1}, X_{2}, \ldots, X_{k}$ so that for each $i,\left(X_{1} \cup \cdots \cup X_{i}\right) \cap X_{i+1}=\operatorname{span}\left(X_{1} \cup \cdots \cup\right.$ $\left.X_{i}\right) \cap \operatorname{span}\left(X_{i+1}\right)$. Then, we have a corollary as follows:

Corollary 4.5. Let $X$ be a nondegenerate subscheme in the category $(*)$ with $e=$ $\operatorname{codim}\left(X, \mathbb{P}^{r+e}\right)$.

(a) (extremal case) $X$ satisfies property $\boldsymbol{N}_{2, e}$ if and only if $X$ is 2-regular, i.e. the linearly joined sequences of $r$-dimensional minimal degree varieties; 
(b) (next-to-extremal case) If $X$ fails property $\boldsymbol{N}_{2, e}$ but satisfies $\boldsymbol{N}_{2, e-1}$, then $X$ is arithmetically Cohen-Macaulay and is of next-to-minimal degree.

Proof. For (a) we can also project $X$ to a hyperquadric $X^{(e-1)}$ similarly. (In this case $X^{(e-1)}$ might be reducible, i.e. a union of two $r$-linear planes.) So $X^{(e-1)}$ is ACM, and from our Depth Theorem 4.1 $X$ is also ACM, eventually 2-regular. We also have a similar result for the case of (b) by the same arguments; $X$ becomes $\mathrm{ACM}$ and of next-to-minimal degree subscheme with $h^{0}\left(\mathcal{J}_{X}(2)\right)=\frac{(e+2)(e-1)}{2}$ in this category.

Remark 4.6. For the 'rigidity' of property $\mathbf{N}_{2, p}$ for $p=e$, D. Eisenbud et al. proved the same theorem for the category of algebraic sets more generally in [EGHP05]. The (geometric) classification of 2-regularity is well known for varieties, and for general algebraic sets it is given in EGHP06. We reprove this rigidity (case (a)) using our inner projection method and for the next-to-extremal case (b) we also get a similar classification for the subschemes in the category $(*)$ (see Question 5.6).

\section{EXAMPLES AND OPEN QUESTIONS}

It seems to be quite natural to find a good inner projection as we move the point $q \in X$ in many aspects. What happens to inner projections from singular points? During the discussions with P. Schenzel, we have the following example.

Example 5.1 (Projection from a singular point, discussion with P. Schenzel). Let us consider a singular surface $X$ in $\mathbb{P}^{14}$ by Segre embedding of a quadric in $\mathbb{P}^{2}$ and a singular quintic rational curve in $\mathbb{P}^{4}$ (note that '-' means "zero" in the Betti table, Table 5.1).

TABle 5.1. A singular surface $X$ in $\mathbb{P}^{14}$ (computed by Singular)

\begin{tabular}{|c|c|c|c|c|c|c|c|c|c|}
\hline & 0 & 1 & 2 & 3 & 4 & 5 & $\cdots$ & $i$ & $\cdots$ \\
\hline 0 & 1 & - & - & - & - & - & $\cdots$ & - & $\cdots$ \\
\hline 1 & - & 70 & 475 & 1605 & 3333 & 4500 & $\cdots$ & $\beta_{i, 1}$ & $\cdots$ \\
\hline 2 & - & - & - & 11 & 100 & 405 & $\cdots$ & $\beta_{i, 2}$ & $\cdots$ \\
\hline 3 & - & - & - & - & - & - & $\ddots$ & $\beta_{i, 3}$ & $\ddots$ \\
\hline
\end{tabular}

We see that $X$ satisfies property $\mathbf{N}_{2,2}$. Now consider inner projections of $X$ from (a) a smooth point and (b) any singular point of $X$ (we can't distinguish the singularities). See Table 5.2. While $X_{q}$ property $\mathbf{N}_{2,1}$ holds in case (a) as our Corollary 3.4 says, in case (b) we still have property $\mathbf{N}_{2,2}$ for $X_{q}$ !

Example 5.2. Let $X$ be the Grassmannian $\mathbb{G}(2,4)$ in $\mathbb{P}^{9}$, a 6 -dimensional del Pezzo variety of degree 5 whose Betti table is shown in Table 5.3 and property $\mathbf{N}_{2,2}$ is satisfied. Since it is homogeneous and covered by lines, we can choose any (smooth) point $q$ in $X$ and a line $\ell$ through $q$ in $X$. Then the projection $X_{q}$ is a complete intersection of two quadrics in $\mathbb{P}^{8}$ (property $\mathbf{N}_{2,1}$ ) and $q^{\prime}=\pi_{q}(\ell$ ) becomes 
TABLE 5.2. (a) from smooth point, (b) from any singular point

$(5.2)(a)$

\begin{tabular}{|c|c|c|c|c|c|}
\hline & 0 & 1 & 2 & 3 & 4 \\
\hline 0 & 1 & - & - & - & - \\
\hline 1 & - & 58 & 351 & 1035 & $\cdots$ \\
\hline 2 & - & - & 1 & 19 & $\cdots$ \\
\hline 3 & - & - & - & - & $\ddots$ \\
\hline
\end{tabular}

(b)

\begin{tabular}{|c|c|c|c|c|c|}
\hline & 0 & 1 & 2 & 3 & 4 \\
\hline 0 & 1 & - & - & - & - \\
\hline 1 & - & 59 & 362 & 1089 & $\cdots$ \\
\hline 2 & - & - & - & 10 & $\cdots$ \\
\hline 3 & - & - & - & - & $\ddots$ \\
\hline
\end{tabular}

TABle 5.3. The Grassmannian $\mathbb{G}(2,4)$ in $\mathbb{P}^{9}$

\begin{tabular}{|c|c|c|c|c|}
\hline & 0 & 1 & 2 & 3 \\
\hline 0 & 1 & - & - & - \\
\hline 1 & - & 5 & 5 & - \\
\hline 2 & - & - & - & 1 \\
\hline
\end{tabular}

a singularity of multiplicity 2 in $X_{q}$. If we project this once more from $q^{\prime}$, then the projected image becomes a quadric hypersurface in $\mathbb{P}^{7}$ still satisfying property $\mathbf{N}_{2,1}$.

Question 5.3 (Inner projection from a singular point). Assume that $X$ is a nondegenerate projective scheme with $\mathbf{N}_{2, p}$. If $q \in X$ is singular, we could expect that the inner projection from $q$ has more complicated aspects, but shows better behavior still satisfying $\mathbf{N}_{2, p}$ in many experimental data. What can happen to the projection from a singular locus in general?

Next, we consider Problem (a) of the introduction in general. Let $X$ be a nondegenerate subscheme with property $\mathbf{N}_{2, p}$. If $\ell$ meets $X$ but is not contained in $X$, then we can regard the projection $\pi_{\ell}$ as the composition of two simple projections from points $q_{1}, q_{2}$. Furthermore, if such an $\ell$ meets $X$ at two smooth points, then $X_{\ell}=\overline{\pi_{\ell}(X \backslash \ell)}$ satisfies property $\mathbf{N}_{2, p-2}$ by our main theorem.

But the case of $\ell \subset X$ cannot be treated simply, because $q_{2}=\pi_{q_{1}}(\ell)$ might be a singular point even if $\pi_{\ell}=\pi_{q_{2}} \circ \pi_{q_{1}}$. In this case, we give an interesting example showing that the Betti numbers of $X_{\ell}=\overline{\pi_{\ell}(X \backslash \ell)}$ are related to the geometry of the line in $X$.

Example 5.4 (Projection from a line inside the variety). Consider the Segre embedding $X=\sigma\left(\mathbb{P}^{2} \times \mathbb{P}^{4}\right)$, 6-fold of degree 15 in $\mathbb{P}^{14}$, having property $\mathbf{N}_{2,3}$ whose Betti table is shown in Table 5.4. In $X$ there are two type of contained lines, socalled $\ell_{1}$ and $\ell_{2}$. If we take $\ell_{1}$ as the line $\sigma(\{p t\} \times \ell)$ in $X$, then the image $X_{\ell_{1}}$ is the intersection of two cones $\left\langle\sigma\left(\mathbb{P}^{1} \times \mathbb{P}^{4}\right), \mathbb{P}^{2}\right\rangle$ and $\left\langle\mathbb{P}^{3}, \sigma\left(\mathbb{P}^{2} \times \mathbb{P}^{2}\right)\right\rangle$, which is 6 -fold of degree 12 in $\mathbb{P}^{12}$ satisfying property $\mathbf{N}_{2,2}$ with the Betti table as in Table 5.5 (a). 
TABLE 5.4. Segre embedding $X=\sigma\left(\mathbb{P}^{2} \times \mathbb{P}^{4}\right)$, 6-fold of degree 15 in $\mathbb{P}^{14}$

\begin{tabular}{|c|c|c|c|c|c|c|c|c|c|}
\hline & 0 & 1 & 2 & 3 & 4 & 5 & 6 & 7 & 8 \\
\hline 0 & 1 & - & - & - & - & - & - & - & - \\
\hline 1 & - & 30 & 120 & 210 & 168 & 50 & - & - & - \\
\hline 2 & - & - & - & - & 50 & 120 & 105 & 40 & 6 \\
\hline
\end{tabular}

On the other hand, in the case of the line $\ell_{2}=\sigma(\ell \times\{p t\}), X_{\ell_{2}}$ is a 6 -dimensional cone $\left\langle\{p t\}, \sigma\left(\mathbb{P}^{2} \times \mathbb{P}^{3}\right)\right\rangle$ of degree 10 in $\mathbb{P}^{12}$ and has its own Betti table as in Table 5.5 (b) with property $\mathbf{N}_{2,3}$. Note that the dimension of the span $\left\langle\bigcup_{q \in \ell_{1}} T_{q} X\right\rangle$ of tangent spaces along $\ell_{1}$ is 8 , but $\operatorname{dim}\left\langle\bigcup_{q \in \ell_{2}} T_{q} X\right\rangle=10$ (i.e. the tangent spaces change more variously along $\ell_{2}$ than $\ell_{1}$ ). So, it is expected that $\ell_{2}$ is geometrically less movable than $\ell_{1}$ inside $X$ and that $X_{\ell_{2}}$ has more linear syzygies.

TABLE 5.5. (a) from a line $\ell_{1}$ of type 1 , (b) from a line $\ell_{2}$ of type 2

\begin{tabular}{|c|c|c|c|c|c|c|c|}
\hline & 0 & 1 & 2 & 3 & 4 & 5 & 6 \\
\hline 0 & 1 & - & - & - & - & - & - \\
\hline 1 & - & 16 & 40 & 30 & 4 & - & - \\
\hline 2 & - & - & - & 20 & 40 & 24 & 5 \\
\hline
\end{tabular}

(b)

\begin{tabular}{|c|c|c|c|c|c|c|c|}
\hline & 0 & 1 & 2 & 3 & 4 & 5 & 6 \\
\hline 0 & 1 & - & - & - & - & - & - \\
\hline 1 & - & 18 & 52 & 60 & 24 & - & - \\
\hline 2 & - & - & - & - & 10 & 12 & 3 \\
\hline
\end{tabular}

Question 5.5 (Inner projection from a subvariety). Let $X$ be a nondegenerate reduced scheme in $\mathbb{P}^{N}$ satisfying property $\mathbf{N}_{2, p}(p>1)$ which is not necessarily linearly normal. Consider the inner projection from a line $\ell \subset X$. Is it true that $\overline{\pi_{\ell}(X \backslash \ell)}$ satisfies at least $\mathbf{N}_{2, p-2}$ ? How does the infinitesimal geometry of $\ell$ in $X$ affect the syzygies of $\overline{\pi_{\ell}(X \backslash \ell)}$ ? More generally, how about the projection from a subvariety $Y$ of $X$ ? The projection from $Y$ is defined by the projection from $\Lambda:=\langle Y\rangle$, the linear span of $Y$ (see BHSS00]). Say $\operatorname{dim} \Lambda=t<p$. Does $X_{\Lambda}$ in $\mathbb{P}^{N-t-1}$ satisfy property $\mathbf{N}_{2, p-t-1}$ in general as raised in the problem list (a) in the introduction?

For the sake of Question [5.5, we expect to need to develop the elimination mapping cone theorem and partial elimination module theory for the multivariate case and to calculate the syzygies of those partial elimination modules by Gröbner basis theory for graded modules. See HK10 for basic settings and some partial results for the bivariate case.

Finally, we have the following question as raised in Remark 4.6

Question 5.6 (Geometric characterization of some 3-regular ACM schemes). We showed in Section 4 that if an $r$-equidimensional, reduced and connected in codimension 1 subscheme $X$ in $\mathbb{P}^{r+e}$ fails property $\mathbf{N}_{2, e}$ but satisfies $\mathbf{N}_{2, e-1}$, then it is an ACM, 3-regular scheme of next-to-minimal degree (i.e. $\operatorname{deg}(X)=\operatorname{codim} X+2$ ) 
with $h^{0}\left(\mathcal{J}_{X}(2)\right)=\frac{(e+2)(e-1)}{2}$. Further, a theorem of L.T. Hoa in Hoa93 gives the complete graded Betti numbers of these schemes as follows:

$(* *) 0 \rightarrow R(-e-2) \rightarrow R^{\beta_{e-2,2}}(-e) \rightarrow R^{\beta_{e-3,2}}(-e+1) \rightarrow \cdots \rightarrow R^{\beta_{0,2}}(-2) \rightarrow I_{X} \rightarrow 0$

where $\beta_{i, 2}=(i+1)\left(\begin{array}{c}e+1 \\ i+2\end{array}\right)-\left(\begin{array}{c}e \\ i\end{array}\right)$ for $0 \leq i \leq e-2$. Thus, just as the characterization of reduced 2-regular projective schemes (see [EGHP06]), among all the equidimensional reduced and connected in codimension 1 subschemes, it would be very interesting to classify or give geometric descriptions for all 3-regular, ACM, and next-to-minimal degree projective schemes whose Betti table is given by $(* *)$.

\section{ACKNOWLEDGEMENTS}

The first author would like to thank Professor Frank-Olaf Schreyer for hosting his visit to Saarbrücken under the KOSEF-DAAD Summer Institute Program and for many valuable comments while preparing this paper. The second author would like to thank Professor B. Sturmfels and M. Brodmann for their useful comments, especially P. Schenzel for valuable discussions and Example 5.1 during their stay at the Korea Institute of Advanced Study (KIAS) and KAIST, Korea in the Summer of 2009. We would also like to thank Professor F. Zak who informed us of Professors A. Alzati and J.C. Sierra's recent paper AS10] related to our paper (see Remark 3.10).

\section{REFERENCES}

[AK11] J. Ahn and S. Kwak, Graded mapping cone theorem, multisecants and syzygies, J. Algebra 331 (2011), 243-262. MR2774656

[AS10] A. Alzati and J.C. Sierra, A bound on the degree of schemes defined by quadratic equations, to appear in 'Forum Mathematicum'.

[Bau95] I. Bauer, Inner projections of algebraic surfaces: a finiteness result, J. Reine Angew. Math. 460 (1995), 1-13. MR.1316568(96a:14059)

[BHSS00] M. Beltrametti, A. Howard, M. Schneider and A. Sommese, Projections from subvarieties, Complex Analysis and Algebraic Geometry (T. Peternell, F-O. Schreyer, eds.), A volume in memory of Michael Schneider, (2000), 71-107. MR.1760873(2001e:14004)

[BP10] M. Brodmann and E. Park, On varieties of almost minimal degree I: Secant loci of rational normal scrolls, J. Pure Appl. Algebra 214 (2010), 2033-2043. MR2645336 (2012a:14120)

[BS07] M. Brodmann and P. Schenzel, Arithmetic properties of projective varieties of almost minimal degree, J. Algebraic Geom. 16 (2007), 347-400. MR2274517 (2008b:14085)

[CC01] A. Calabri and C. Ciliberto, On special projections of varieties: epitome to a theorem of Beniamino Segre, Adv. Geom. 1 (2001), no. 1, 97-106. MR1823955 (2002b:14064)

[CKK06] Y. Choi, S. Kwak and P-L. Kang, Higher linear syzygies of inner projections, J. Algebra 305 (2006), 859-876. MR2266857(2007k:14008)

[CKP08] Y. Choi, S. Kwak and E. Park, On syzygies of non-complete embedding of projective varieties, Math. Zeitschrift 258, no. 2 (2008), 463-475. MR.2357647 (2009i:13023)

[EGHP05] D. Eisenbud, M. Green, K. Hulek and S. Popescu, Restriction linear syzygies: algebra and geometry, Compositio Math. 141 (2005), 1460-1478. MR2188445 (2006m:14072)

[EGHP06] D. Eisenbud, M. Green, K. Hulek and S. Popescu, Small schemes and varieties of minimal degree, Amer. J. Math. 128 (2006), no. 6, 1363-1389. MR2275024 (2007j:14078)

[EHU06] D. Eisenbud, C. Huneke, and B. Ulrich, The regularity of Tor and graded Betti numbers, Amer. J. Math. 128 (2006), no. 3, 573-605. MR.2230917 (2007b:13027)

[Fuj90] T. Fujita, Classification theories of polarized varieties, Cambridge University Press, Cambridge, 1990. MR1162108 (93e:14009)

[FCV99] H. Flenner, L. O'Carroll, and W. Vogel, Joins and intersections, Springer-Verlag, Berlin, 1999. MR1724388(2001b:14010) 
[Gre84] M. Green, Koszul cohomology and the geometry of projective varieties, J. Differential Geom. 19 (1984), 125-171. MR739785 (85e:14022)

[Gre98] M. Green, Generic Initial Ideals, in Six lectures on Commutative Algebra, (Elias J., Giral J.M., Miró-Roig, R.M., Zarzuela S., eds.), Progress in Mathematics 166, Birkhäuser, 1998, 119-186. MR:1648665 (99m:13040)

[GL88] M. Green and R. Lazarsfeld, Some results on the syzygies of finite sets and algebraic curves, Compositio Math. 67 (1988), no. 3, 301-314. MR959214 (90d:14034)

[HK10] K. Han and S. Kwak, Projections from lines: algebraic and geometric properties, in preparation.

[Hart77] R. Hartshorne, Algebraic geometry, Graduate Texts in Math., Springer-Verlag, New York, 1977. MR 0463157(57:3116)

[Hoa93] L.T. Hoa, On minimal free resolutions of projective varieties of degree = codimension +2, J. Pure Appl. Algebra, 87 (1993), 241-250. MR.1228155 (94e:13024)

[Katz93] S. Katz, Arithmetically Cohen-Macaulay curves cut out by quadrics, Computational algebraic geometry and commutative algebra (Cortona, 1991), 257-263, Sympos. Math., XXXIV, Cambridge Univ. Press, Cambridge, 1993. MR.1253994 (94k:14021)

[KP05] S. Kwak and E. Park, Some effects of property $\mathrm{N}_{p}$ on the higher normality and defining equations of nonlinearly normal varieties, J. Reine Angew. Math. 582 (2005), 87-105. MR2139712 (2006d:14009)

[OP01] G. Ottaviani and R. Paoletti, Syzygies of Veronese embeddings, Compositio Math., 125 (2001), 31-37. MR 1818055 (2002g:13023)

[Park08] E. Park, On secant loci and simple linear projections of some projective varieties, preprint

[Reid00] M. Reid, Graded rings and birational geometry, in Proc. of Algebraic Geometry Symposium (Kinosaki, Oct. 2000), K. Ohno (Ed.), 1-72.

[Seg36] B. Segre, On the locus of points from which an algebraic variety is projected multiply, Proc. Phys.-Math. Soc. Japan Ser. III, 18 (1936), 425-426.

[Som79] A. Sommese, Hyperplane sections of projective surfaces I, The adjunction mapping, Duke Math. J. 46 (1979), no. 2, 377-401. MR534057 (82f:14033)

[Zak99] F. L. Zak, Projective invariants of quadratic embedding, Math. Ann. 313 (1999), 507545. MR1678545 (2000b:14071)

Department of Mathematics, Korea Advanced Institute of Science and Technology, 373-1 Gusung-dong, Yusung-Gu, Daejeon, Korea

E-mail address: han.kangjin@kaist.ac.kr

Current address: School of Mathematics, Korea Institute for Advanced Study, Seoul 130-722, Korea

E-mail address: kangjin.han@kias.re.kr

Department of Mathematics, Korea Advanced Institute of Science and Technology, 373-1 Gusung-Dong, Yusung-Gu, Daejeon, Korea

E-mail address: skwak@kaist.ac.kr 